\title{
Strongyloidiasis in personnel of the Regional Assistance Mission to Solomon Islands (RAMSI)
}

\author{
David A Pattison and Richard Speare
}

S trongyloides stercoralis is a soil-transmitted parasitic gastrointestinal nematode that can persist for years in the human intestine through the production of autoinfective larvae. ${ }^{1}$ Patients at risk of strongyloidiasis should be screened to prevent hyperinfection syndrome before they are given chemotherapy or other immunosuppressant drugs (including corticosteroids). In this syndrome, autoinfective larvae disseminate in large numbers from the gastrointestinal tract, leading to superimposed bacterial meningitis and septicaemia; it has a mortality rate of up to $87 \% .^{2}$

Although strongyloidiasis occurs in Papua New Guinea, ${ }^{3}$ Fiji ${ }^{4}$ and Vanuatu, ${ }^{5}$ it has not been reported in the Solomon Islands. This may be because faecal microscopy is not routinely performed at the National Referral Hospital in Honiara, and serology testing is not available in the Solomon Islands (Dr Tenneth Dalipanda, Chief Executive Officer and Medical Superintendent, National Referral Hospital, Honiara, Solomon Islands, personal communication).

The only published survey of soil-transmitted helminths in the Solomon Islands was conducted in a semi-urban environment, and reported that $40.7 \%-45 \%$ of children tested were infected with a soiltransmitted helminth — Ascaris lumbricoides, hookworm or Trichuris trichiura. ${ }^{6}$

Protracted ethnic tensions in the Solomon Islands (1998-2003) resulted in the establishment of the Regional Assistance Mission to Solomon Islands (RAMSI). Member countries of the Pacific Islands Forum, under Australia's leadership, established RAMSI in 2003 with the aim of restoring law and order and assisting the Solomon Islands' economic recovery. Participating nations have sent several thousand military personnel, police officers and civilian advisers to the Solomon Islands; most are from Australia and New Zealand. Until recently, before returning to their country of origin, RAMSI personnel received mebendazole, which is ineffective against $S$. stercoralis. ${ }^{7}$

The RAMSI Medical Facility in Honiara is contracted by the Australian Government to provide comprehensive health care

\section{ABSTRACT}

Objective: To investigate the first reported cases of strongyloidiasis in the Solomon Islands, and to establish whether this disease poses a risk to personnel of the Regional Assistance Mission to Solomon Islands (RAMSI).

Design, setting and participants: Retrospective review of the pathology database of the RAMSI Medical Facility in Honiara, Solomon Islands, for the period 1 July 2006 30 September 2007.

Main outcome measures: Number and clinical features of confirmed cases of Strongyloides stercoralis infestation, as diagnosed by serological tests or faecal microscopy.

Results: Fourteen confirmed cases of strongyloidiasis in previously healthy RAMSI participants were identified. Of 13 patients with notes available, symptoms documented at presentation included epigastric pain (10 patients), diarrhoea (7) and urticaria (4). Clinical disease in all patients responded to oral antihelminthic therapy (albendazole or ivermectin).

Conclusions: Strongyloidiasis is endemic in the Solomon Islands and a risk for RAMSI personnel. Australian medical professionals should be aware of this potentially fatal and lifelong infestation, particularly the importance of an occupation history, appropriate diagnostic tests, effective treatment and adequate follow-up to document cure. We recommend implementation of a postdeployment screening program for strongyloidiasis.

MJA 2008; 189: 203-206

for RAMSI personnel, who are estimated to number around 1000 at any given time.

At the Medical Facility, we diagnosed strongyloidiasis in several personnel. This led to concern for undiagnosed personnel who return home to non-endemic regions, where the awareness of the implications of prior exposure is limited. Prescription of chemotherapy, corticosteroids or other immunosuppressive medications without appropriate screening and treatment in this cohort would be of particular concern. Therefore, we undertook a retrospective review of the RAMSI Medical Facility pathology database to determine the epidemiology of strongyloidiasis in RAMSI personnel and the need for a postdeployment screening program.

\section{METHODS}

Cases of $S$. stercoralis infestation were diagnosed based on faecal microscopy or serology.

Stool samples were examined via direct microscopy of faeces concentrate (Mini Parasep faecal parasite concentration kit; DiaSys, Wokingham, Berkshire, UK) by an onsite medical scientist. S. stercoralis can be differentiated from Strongyloides fuelleborni (which causes limited disease in humans) based on the presence of larvae and not eggs in the faeces. ${ }^{8}$

Serological tests were performed at the Queensland Medical Laboratory, Brisbane, using enzyme-linked immunosorbent assay (ELISA). Optical density was categorised as negative ( $<0.9$ optical density units [ODU]), equivocal (0.9-1.2 ODU), or positive (> 1.2 ODU).

We retrospectively reviewed the pathology database of the RAMSI Medical Facility for the period 1 July 2006 - 30 September 2007. We searched the database for the results of faecal examinations and Strongyloides serological tests. The medical records of patients with positive results were reviewed, if available.

\section{RESULTS}

We found 168 faecal examination results and 34 Strongyloides serological test results; some patients had received both tests. We identified 14 patients with S. stercoralis infestation: 11 men and three women (average age, 36 years). Only one patient was deployed from an area with high 


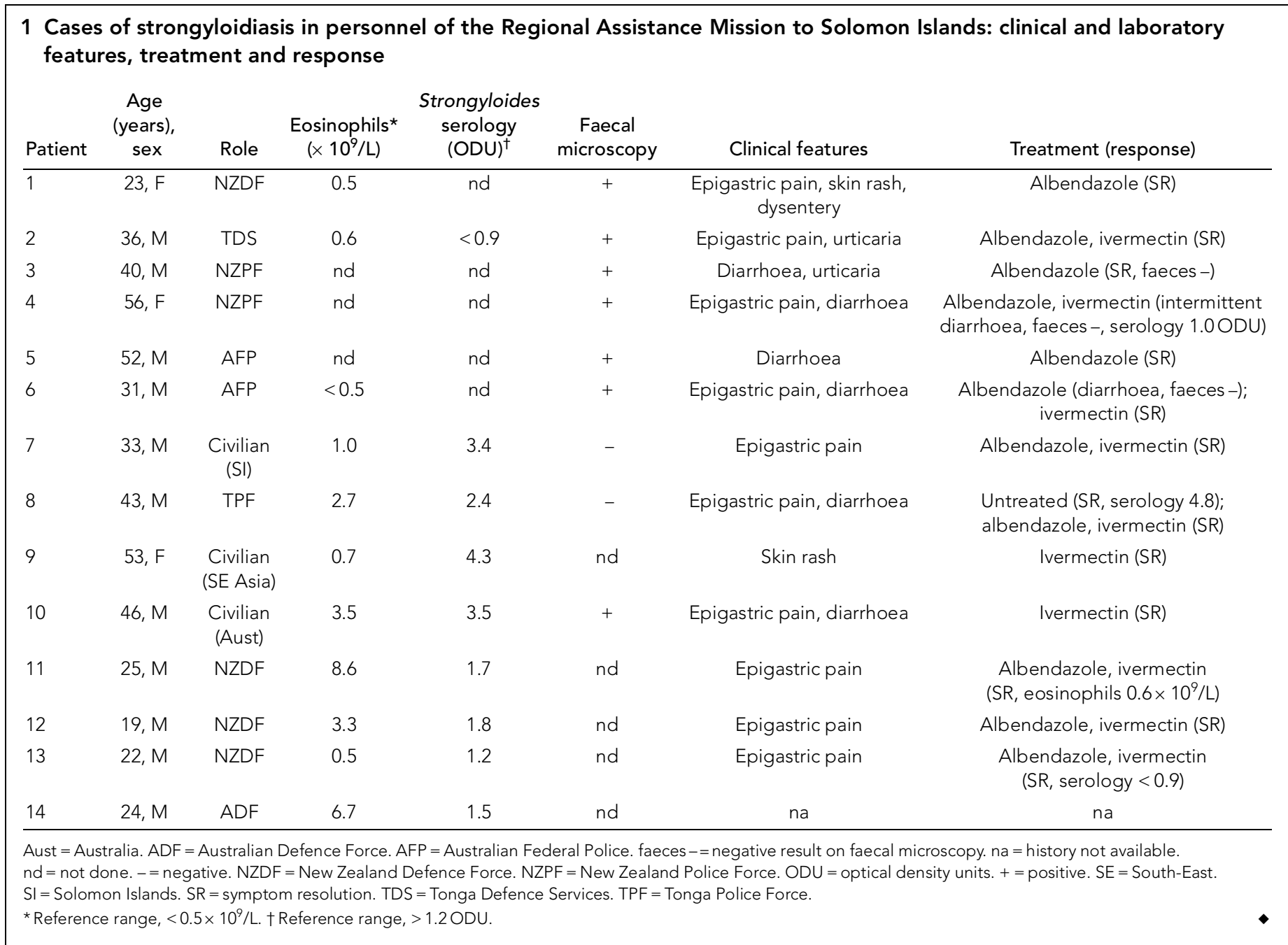

endemicity (Patient 9, from South-East Asia) (Box 1). S. fuelleborni infestation was not observed.

Thirteen of the 14 patients had case notes available for review. Among these, epigastric pain exacerbated by eating was the most common symptom (10/13; 77\%). Diarrhoea and urticaria or other skin manifestations were reported in 54\% (7/13) and $31 \%$ (4/13) of individuals, respectively (Box 1).

Importantly, we found that the symptoms of strongyloidiasis were frequently misinterpreted. Epigastric pain was misdiagnosed as gastro-oesophageal reflux, and was thus treated unsuccessfully with proton-pump inhibitors. Two patients with urticarial rash were diagnosed as having insect bites and were treated with corticosteroids. Notably, Patient 1 received $40 \mathrm{mg}$ methylprednisolone intramuscularly, and developed lower abdominal pain, back pain and dysentery 2 days later, which necessitated hospital admission. Faecal microscopy revealed $S$. stercoralis larvae, and prompt treatment with albendazole led to resolution of symptoms.

We cannot report detailed follow-up information due to the limited duration of deployments and difficulty accessing patients' records upon return to their countries of origin. However, Box 1 details the treatments given after strongyloidiasis was diagnosed, and the initial clinical response. The standard treatments used were oral albendazole $400 \mathrm{mg}$ twice daily for 3 days or a single dose of oral ivermectin $200 \mu \mathrm{g} / \mathrm{kg}$.

\section{DISCUSSION}

This retrospective audit identifies the Solomon Islands as an S. stercoralis-endemic region and RAMSI personnel as being at risk of infestation.

A strength of this study was the presence of a facility in the Solomon Islands capable of performing microscopy and with access to serological testing. Many of these cases would not have been diagnosed without serological testing.

The literature provides limited data on the symptoms of acute strongyloidiasis. Our data on presenting symptoms are useful and serve to remind clinicians to consider strongyloidiasis as a differential diagnosis in all symptomatic returned RAMSI personnel, particularly those presenting with epigastric pain, diarrhoea or urticaria. Larva currens - a rapidly progressing rash caused by migration of the larvae and pathognomonic of strongyloidiasis - was not documented in this case series. This is consistent with previous reports that suggest it is rarely seen in acute infestations. ${ }^{9}$

Given the nature of the study, and fluctuation in numbers and frequent rotation of RAMSI personnel, we cannot estimate disease incidence. However, it is likely that our audit underestimated the number of 


\section{Recommendations for management of strongyloidiasis}

\section{General principles}

- Assume lifelong infestation unless eradication is proven

- Screen high-risk groups before immunosuppression (especially with corticosteroids) to prevent hyperinfection syndrome

High-risk groups

- Indigenous Australians

- Immigrants*

- Returned travellers*

- Expatriates: military, police and civilians (including Regional Assistance Mission to Solomon Islands personnel)*

Diagnosis

- Full blood count (to detect eosinophilia)

- Serological tests

- Faecal microscopy ( \pm special techniques to increase sensitivity)

Treatment

- A single dose of ivermectin $200 \mu \mathrm{g} / \mathrm{kg}$

- Three-monthly follow-up (eosinophilia/ serology)

- Re-treatment if positive

* From or who worked in endemic tropical regions.

RAMSI personnel infected during the study period because:

- strongyloidiasis can be asymptomatic; ${ }^{10}$

- symptomatic individuals might have failed to seek medical attention or been treated empirically with antihelminthic therapy;

- some personnel might have developed symptoms only after leaving the Solomon Islands; and

- diagnostic tests have significant limitations. A recent study suggests the Strongyloides ELISA is significantly less sensitive in returned travellers (patients with acute infestation, similar to our cohort) compared with immigrants with previous chronic exposure (73\% v $98 \%$; $P<0.001) .{ }^{11}$ Further, the time necessary for seroconversion in humans is unknown. ${ }^{12}$ Thus, false-negative results may be obtained in patients tested before seroconversion. Faecal microscopy has low sensitivity in chronic uncomplicated strongyloidiasis, ${ }^{10}$ and its sensitivity in acute strongyloidiasis is not clearly defined. However, diagnostic accuracy can be improved by examining multiple stool samples or using Strongyloides agar-plate culture. $^{13}$
It is vital to identify and treat individuals with strongyloidiasis before immunosuppression in order to minimise the risk of hyperinfection syndrome. Our study highlights this, as systemic corticosteroids were used to treat urticarial skin rash in two of our 14 patients, with adverse consequences; corticosteroid treatment was also the precipitant of fatal disseminated strongyloidiasis in a case reported in 2001. ${ }^{14}$

The diagnosis of strongyloidiasis before immunosuppression requires a high index of suspicion. As Australian-led interventions in the Asia-Pacific region are likely to increase, an increasing number of Australians will be exposed to S. stercoralis. Thus, it is evident that all patients being considered for immunosuppressive therapy must have an occupational history taken. If this reveals a geographic risk factor for strongyloidiasis, appropriate testing and treatment (if positive) must be instituted before immunosuppression.

Accordingly, we propose a postdeployment screening program comprising a full blood count (to detect eosinophilia), Strongyloides serology and microscopy, and agar-plate culture of a stool specimen. The absence of a "gold-standard" test mandates the use of a combined diagnostic approach. These tests could be performed 3 months after departure from the Solomon Islands, coinciding with screening for exposure to bloodborne viruses and tuberculosis to maximise compliance with follow-up.

Our incomplete follow-up data prevent assessment of treatment efficacy. However, ivermectin is currently considered first-line treatment for uncomplicated S. stercoralis infestations due to its high efficacy ( $83 \%$ microscopic clearance after 3 months). ${ }^{15,16}$ Although albendazole ( $400 \mathrm{mg}$ twice daily for 3 days) is less efficacious ( $75 \%$ microscopic clearance after 6 months), ${ }^{17}$ its broader antihelminthic activity (especially against hookworm) makes it more suitable for empirical treatment. ${ }^{18,19}$ It has recently replaced mebendazole as the postdeployment antihelminthic eradication therapy used by RAMSI.

Follow-up serological testing to document eradication is a necessary part of a screening program, as strongyloidiasis is considered a lifelong infestation until proven eradicated. The case of a Laotian immigrant to Australia who died from Strongyloides hyperinfection caused by inadvertent immunosuppression a year after treatment with albendazole ${ }^{14}$ supports this component of the proposed screening program (Box 2).
Staff inductions have been changed to include information about the lifelong nature of strongyloidiasis infestation and its potentially fatal complications. RAMSI personnel are advised to avoid skin exposure to soil that may have been contaminated by human faeces, principally by wearing appropriate footwear. Australian medical professionals should be aware that strongyloidiasis is endemic in the Solomon Islands and that RAMSI personnel are at risk of infestation. A postdeployment screening program is recommended for all RAMSI personnel.

\section{ACKNOWLEDGEMENTS}

We thank Dr Orla Morrissey for reviewing the manuscript, Dr Ross Fountain and Lance Corporal Justine O'Dwyer from the New Zealand Defence Force for patient follow-up, and Mr Robert Dwyer (medical scientist, RAMSI Medical Facility, Honiara) for input on parasitology.

\section{COMPETING INTERESTS}

None identified.

\section{AUTHOR DETAILS}

David A Pattison, MB BS, Medical Officer ${ }^{1}$ Richard Speare, MB BS, PhD, FAFPHM, Director $^{2}$

1 RAMSI Medical Facility, Aspen Medical, Honiara, Solomon Islands.

2 Anton Breinl Centre for Public Health and Tropical Medicine, James Cook University, Townsville, QLD.

Correspondence: david.pattison@jcu.edu.au

\section{REFERENCES}

1 Grove DI. Human strongyloidiasis. Adv Parasitol 1996; 38: 251-309.

2 Link K, Orenstein R. Bacterial complications of strongyloidiasis: Streptococcus bovis meningitis. South Med J 1999; 92: 728-731.

3 Shann F, Biddulph J, Vince J. Paediatrics for doctors in Papua New Guinea: a guide for doctors providing health services for children. 2nd ed. Madang: Papua New Guinea Department of Health, 2003: 356.

4 Thomas M, Woodfield G, Moses C, et al. Soiltransmitted helminth infection, skin infection, anaemia, and growth retardation in schoolchildren of Taveuni Island, Fiji. N Z Med J 2005; 118: U1492.

5 Einsiedel L, Spelman D. Strongyloides stercoralis: risks posed to immigrant patients in an Australian tertiary referral centre. Intern Med J 2006; 36: 632-637.

6 Hughes RG, Sharp DS, Hughes MC, et al. Environmental influences on helminthiasis and nutritional status among Pacific schoolchildren. Int J Environ Health Res 2004; 14: 163-177.

7 Keystone JS, Murdoch JK. Mebendazole. Ann Intern Med 1979; 91: 582-586.

8 Speare R. Identification of species of Strongyloides. In: Grove DI, editor. Strongyloidiasis: an 
important roundworm infection of man. London: Taylor Francis, 1989: 11-82.

9 Gill GV, Welch E, Bailey JW, et al. Chronic Strongyloides stercoralis infection in former British Far East prisoners of war. QJM 2004; 97 : 789-795.

10 Siddiqui AA, Berk SL. Diagnosis of Strongyloides stercoralis infection. Clin Infect Dis 2001; 33: 1040-1047.

11 Sudarshi S, Stumpfle R, Armstrong $M$, et al. Clinical presentation and diagnostic sensitivity of laboratory tests for Strongyloides stercoralis in travellers compared with immigrants in a non-endemic country. Trop Med Int Health 2003; 8: 728-732.

12 Speare R, Durrheim DN. Strongyloides serology - useful for diagnosis and management of strongyloidiasis in rural Indigenous populations, but important gaps in knowledge remain. Rural Remote Health 2004; 4: 264.

13 Koga K, Kasuya S, Khamboonruang C, et al. A modified agar plate method for detection of Strongyloides stercoralis. Am J Trop Med Hyg 1991; 45: 518-521.

14 Lim L, Biggs BA. Fatal disseminated strongyloidiasis in a previously treated patient. Med $J$ Aust 2001; 174: 355-356.

15 Datry A, Hilmarsdottir I, Mayorga-Sagastume R, et al. Treatment of Strongyloides stercoralis infection with ivermectin compared with albendazole: results of an open study of 60 cases. Trans R Soc Trop Med Hyg 1994; 88: 344-355.

16 Marti H, Haji HJ, Savioli L, et al. A comparative trial of single dose ivermectin versus three days of albendazole for treatment of Strongyloides stercoralis and other soil-transmitted helminth infections in children. Am J Trop Med Hyg 1996; 55: 477-481.

17 Archibald LK, Beeching NJ, Gill GV, et al. Albendazole is effective treatment for chronic strongyloidiasis. Q J Med 1993; 86: 191-195.

18 Bailey MS, Thomas R, Green AD, et al. Helminth infections in British troops following an operation in Sierra Leone. Trans $R$ Soc Trop Med Hyg 2006; 100: 842-846.

19 Muennig P, Pallin D, Sell RL, et al. The cost effectiveness of strategies for the treatment of intestinal parasites in immigrants. N Engl J Med 1999; 340: 773-779.

(Received 12 Nov 2007, accepted 2 Mar 2008) 